\title{
A Variational Expansion for the Free Energy of a Bosonic System
}

\author{
Wen-Fa Lu ${ }^{a, b, c}$, Sang Koo You ${ }^{d}$, Jino Bak ${ }^{a, c}$, Chul Koo Kim ${ }^{a, c}$ and Kyun Nahme \\ ${ }^{a}$ Institute of Physics and Applied Physics, Yonsei University, Seoul 120-749, Korea \\ ${ }^{b}$ Department of Physics, and Institute for Theorectical Physics, \\ Shanghai Jiao Tong University, Shanghai 200030, The People's Republic of China* \\ ${ }^{c}$ Center for Strongly Correlated Materials Research, Seoul National University, \\ Seoul 151-742, Korea \\ ${ }^{d}$ Max-Planck Institute for the Physics of Complex Systems, D-01187 Dreaden, Germany \\ e Department of Physics, Yonsei University, Wonju 220-710, Korea
}

\begin{abstract}
In this paper, a variational perturbation scheme for nonrelativistic many-Fermion systems is generalized to a Bosonic system. By calculating the free energy of an anharmonic oscillator model, we investigated this variational expansion scheme for its efficiency. Using the modified Feynman rules for the diagrams, we obtained the analytical expression of the free energy up to the fourth order. Our numerical results at various orders are compared with the exact and other relevant results.
\end{abstract}

\section{INTRODUCTION}

For calculating the free energy of a system, there exist usually two basic methods, the conventional perturbative and the variational methods [1]. In fact, these two methods are standard in calculations of many physics problems. However, it is well known that the former is useful only for small perturbing potentials, whereas the latter lacks systematic schemes to control its accuracy, albeit it is valid for any potential. In order to overcome these difficulties and improve the variational method, a variational perturbation idea of properly combining the two methods was pioneered by Koehler in lattice dynamics [2] and Seznec and Zinn-Justin [3] on an anharmonic osillator decades ago.

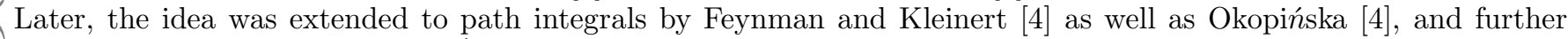
developed by other authors 55 . 7 . Very recently, three of the authors (You, Kim and Nahm) and their collaborator presented a variational perturbation scheme for a nonrelativistic many-Fermion system in the functional integral formalism [8]. In order to test the efficiency of the method, Ref. [8] provided a model numerical calculation at the zero temperature up to the second order. Obviously, this test is limited, and a more wide investigation is necessary for the variational perturbation scheme.

Actually, the scheme in Ref. [8] is a Taylor series expansion based on the variational result obtained in the spirit of Feynman variational principle [1]. This scheme can improve the variational method if used judicially. We note that there exists no work performing the same scheme for Bosonic systems. Although Okopińska [9] and Krzyweck [6] established two kinds of expansion schemes, the optimized expansions and the cumulant expansions respectively, whose lowest order results are the variational result, they are really not based on the variational result because the variational procedure was performed at the truncated order. Further, improvement to the variational method is notoriously difficult, and different schemes will have their own advantages over others. So it is worthwhile generalizing the scheme in Ref. [8] to Bosonic systems.

For the above two purposes, an anharmonic oscillator will be an effective laboratory. For a one-dimensional anharmonic oscillator, the Hamiltonian is

$$
H(t)=\frac{1}{2 m} p^{2}+\frac{1}{2} m \omega^{2} x^{2}(t)+\lambda x^{4}(t) .
$$

Here, $x$ is the space position, $p$ the momentum, $t$ the time, and $m, \omega$ as well as $\lambda$ are the mass, frequency and coupling strength, respectively. Such an anharmonic oscillator is probably the simplest model which does not have exact analytic solution. Its exact free energy was obtained numerically 10,6]. Moreover, there exist many approximation methods to calculate its free energy [4,6,9,11]. All of these make Eq.(1) an ideal candidate for our aim. Therefore, in this paper, we will generalize the scheme in Ref. [8], taking the simple anharmonic oscillator, Eq.(1), as a laboratory and investigate the Taylor expansion scheme on the free energy based on the variational result obtained from the Feynman variational principle [1].

\footnotetext{
*permanent address,E-mail: wenfalu@online.sh.cn

${ }^{1}$ Here we are far from exausting the relevant literature.
} 
Simultaneously, the anharmonic oscillator itself is useful in chemical physics [12 and many physics problems, such as thermal expansion, phonon softening and structural phase transitions [13]. Although its free energy was calculated by using many methods, their numerical (approximate and exact) results ${ }^{2}$ were focused mainly on the case of the reduced temperature $T$ less than 1, except for Ref. [10] which provided accurate results at moderate $T$. Therefore, we also test whether our scheme can produce reliable results at moderate temperature range.

We will work within the functional integral formalism [14]. Next section generalizes the scheme in Ref. [8] to calculate the free energy of a system with the potential $V(x)$. In Sect.III, we carry out the scheme on the anharmonic oscillator, Eq.(1). Sect.IV presents calculations of the free energy for Eq.(1) up to the fourth order and makes comparisons with the exact, and various approximate results, such as, variational, cumulant-expansion and optimized-expansion results. Conclusions are given in Sect.V.

\section{A VARIATIONAL EXPASION OF FREE ENERGIES FOR BOSONIC SYSTEMS}

For a system with the Hamiltonian $H(t)=\frac{1}{2 m} p^{2}+V[x(t)]$ with $V[x(t)]=\frac{1}{2} m \omega^{2} x^{2}(t)+V_{I}[x(t)]$, the generating functional is [14] (1990)

$$
Z[J]=\int_{x(0)=x(\beta)} \mathcal{D}[x(\tau)] \exp \left\{-\int_{0}^{\beta}\left[\frac{1}{2} m x(\tau)\left(-\partial_{\tau}^{2}\right) x(\tau)+V[x(\tau)]-J(\tau) x(\tau)\right] d \tau\right\},
$$

where $\tau=i t$ is the imaginary time, $\partial_{\tau} \equiv \frac{\partial}{\partial \tau}, \beta=\frac{1}{\kappa T}$ with $\kappa$ the Boltzmann constant (we will consider it as unity for convenience) and $T$ the temperature. $J$ is an arbitrary external source and the symbol $\mathcal{D}[x(\tau)]$ represents the functional measure. In Eq. $(2), x(0)=x(\beta)$ means that the functional integral should be executed over all the closed paths 14] (1990). To introduce a variational parameter $\Omega$, one can rewrite the classical action functional $S[x]$ in the Euclidean space-time in Eq.(2) as [3,6, 9 ]

$$
\begin{aligned}
S[x, J]= & \int_{0}^{\beta}\left[\frac{1}{2} m x(\tau)\left(-\partial_{\tau}^{2}+\Omega^{2}\right) x(\tau)-J(\tau) x(\tau)-\frac{1}{2} m \Omega^{2} x^{2}(\tau)+V[x(\tau)]\right] d \tau \\
& \equiv S_{0}[x, J]+S_{D}[x]
\end{aligned}
$$

with $S_{0}[x, J]=\int_{0}^{\beta}\left[\frac{1}{2} m x(\tau)\left(-\partial_{\tau}^{2}+\Omega^{2}\right) x(\tau)-J(\tau) x(\tau)\right] d \tau$ and $S_{D}[x]=\int_{0}^{\beta}\left[-\frac{1}{2} m \Omega^{2} x^{2}(\tau)+V[x(\tau)]\right] d \tau$. Thus, $Z[J]$ can be rewritten as

$$
\begin{aligned}
Z[J] & =\int_{x(0)=x(\beta)} \mathcal{D}[x(\tau)] \exp \left\{-S_{0}[x, J]-S_{D}[x]\right\} \\
& =\exp \left\{-\int_{0}^{\beta}\left[-\frac{1}{2} m \Omega^{2} \delta_{J_{\tau}}^{2}+V\left[\delta_{J_{\tau}}\right]\right] d \tau\right\} \int_{x(0)=x(\beta)} \mathcal{D}[x(\tau)] \exp \left\{-S_{0}[x, J]\right\} \\
& =\left(\operatorname{Det}\left(G^{-1}\right)\right)^{-\frac{1}{2}} \exp \left\{-\int_{0}^{\beta}\left[-\frac{1}{2} m\left(\Omega^{2}-\omega^{2}\right) \delta_{J_{\tau}}^{2}+V_{I}\left[\delta_{J_{\tau}}\right]\right] d \tau\right\} \exp \left\{\frac{1}{2} J_{\tau} G_{\tau \tau^{\prime}} J_{\tau^{\prime}} d \tau d \tau^{\prime}\right\},
\end{aligned}
$$

where, $J_{\tau}=J(\tau), \delta_{J_{\tau}} \equiv \frac{\delta}{\delta J_{\tau}}, G^{-1}$ represents the operator $-\partial_{\tau}^{2}+\Omega^{2}$ with the propagator $G_{\tau \tau^{\prime}}=G\left(\tau, \tau^{\prime}\right)$ and $D e t$ means the determinant. In the third equation of Eq.(4), we have carried out the Gaussian functional integration [14 (1990). Note that $G_{\tau \tau^{\prime}}$ can be expanded into a series owing to the closed path requirement in the functional integral, Eq.(2) [14,15]. To calculate the free energy, we express the partition function $Z \equiv Z[J=0]$ in the following form,

$$
\begin{aligned}
Z & =\int_{x(0)=x(\beta)} \mathcal{D}[x(\tau)] \exp \left\{-S_{0}[x, J=0]\right\} \frac{\int_{x(0)=x(\beta)} \mathcal{D}[x(\tau)] \exp \left\{-S_{0}[x, J=0]-S_{D}[x]\right\}}{\int_{x(0)=x(\beta)} \mathcal{D}[x(\tau)] \exp \left\{-S_{0}[x, J=0]\right\}} \\
& =\left(\operatorname{Det}\left(G^{-1}\right)\right)^{-\frac{1}{2}}<\exp \left\{-\int_{0}^{\beta}\left[-\frac{1}{2} m\left(\Omega^{2}-\omega^{2}\right) \delta_{J_{\tau}}^{2}+V_{I}\left[\delta_{J_{\tau}}\right]\right] d \tau\right\}>_{G} \\
& =\left(\operatorname{Det}\left(G^{-1}\right)\right)^{-\frac{1}{2}} \exp \left\{-\int_{0}^{\beta}<-\frac{1}{2} m\left(\Omega^{2}-\omega^{2}\right) \delta_{J_{\tau}}^{2}+V_{I}\left[\delta_{J_{\tau}}\right]>_{G} d \tau\right\}
\end{aligned}
$$

\footnotetext{
${ }^{2}$ In this paper, an anharmonic oscillator does not include the double-well potential case.
} 


$$
\begin{aligned}
\cdot<\exp \left\{-\int_{0}^{\beta}\left[-\frac{1}{2} m\left(\Omega^{2}-\omega^{2}\right) \delta_{J_{\tau}}^{2}+V_{I}\left[\delta_{J_{\tau}}\right]\right.\right. \\
\left.\left.-<-\frac{1}{2} m\left(\Omega^{2}-\omega^{2}\right) \delta_{J_{\tau}}^{2}+V_{I}\left[\delta_{J_{\tau}}\right]>_{G}\right] d \tau\right\}>_{G} .
\end{aligned}
$$

Here, we have used the following notation and relation

$$
\begin{aligned}
<O[x]>_{G} & \equiv \frac{\int_{x(0)=x(\beta)} \mathcal{D}[x(\tau)] O[x] \exp \left\{-\int_{0}^{\beta} \frac{1}{2} m x(\tau)\left(-\partial_{\tau}^{2}+\Omega^{2}\right) x(\tau) d \tau\right\}}{\int_{x(0)=x(\beta)} \mathcal{D}[x(\tau)] \exp \left\{-\int_{0}^{\beta} \frac{1}{2} m x(\tau)\left(-\partial_{\tau}^{2}+\Omega^{2}\right) x(\tau) d \tau\right\}} \\
& =\left.O\left[\delta_{J}\right] \exp \left\{\frac{1}{2} J_{\tau} G_{\tau \tau^{\prime}} J_{\tau^{\prime}} d \tau d \tau^{\prime}\right\}\right|_{J=0} \equiv<O\left[\delta_{J}\right]>_{G} .
\end{aligned}
$$

Obviously, when $V_{I}[x(t)]$ is not zero, it will be impossible to obtain analytically exact partition function and, hence, one has to design some scheme to produce an approximate solution. For the case of small $V_{I}$, one can take $\Omega=\omega$, make a Taylor series expansion to the exponential in Eq.(5) and then truncate the series at some order to approximate $Z$. This is just the conventional perturbation theory and the propergator $G_{\tau \tau^{\prime}}$ is the bare propagator. When $V_{I}$ is not so small, the above perturbation method is valid no longer. In such a case, an effective alternative is the variational method which is based on the Fenyman variational principle. In the following, we briefly introduce the variational method.

Exploiting the Jensen's inequality [1],8], one can have

$$
\begin{gathered}
\quad \exp \left\{-\int_{0}^{\beta}\left[-\frac{1}{2} m\left(\Omega^{2}-\omega^{2}\right) \delta_{J_{\tau}}^{2}+V_{I}\left[\delta_{J_{\tau}}\right]\right] d \tau\right\}>_{G} \\
\geq \exp \left\{-\int_{0}^{\beta}<-\frac{1}{2} m\left(\Omega^{2}-\omega^{2}\right) \delta_{J_{\tau}}^{2}+V_{I}\left[\delta_{J_{\tau}}\right]>_{G} d \tau\right\} .
\end{gathered}
$$

Substituting the above equation into Eq.(6) leads to a relation for the lower limit of the partition function, i.e.,

$$
Z \geq\left(\operatorname{Det}\left(G^{-1}\right)\right)^{-\frac{1}{2}} \exp \left\{-\int_{0}^{\beta}<-\frac{1}{2} m\left(\Omega^{2}-\omega^{2}\right) \delta_{J_{\tau}}^{2}+V_{I}\left[\delta_{J_{\tau}}\right]>_{G} d \tau\right\}
$$

Hence, the free energy is

$$
F=-\frac{1}{\beta} \ln (Z) \leq \frac{1}{2 \beta} \ln \left(\operatorname{Det}\left(G^{-1}\right)\right)+\frac{1}{\beta} \int_{0}^{\beta}<-\frac{1}{2} m\left(\Omega^{2}-\omega^{2}\right) \delta_{J_{\tau}}^{2}+V_{I}\left[\delta_{J_{\tau}}\right]>_{G} d \tau \equiv \bar{F} .
$$

Obviously, making $\bar{F}$ of the last equation the absolute minimum will lead to a minimum upper limit of the free energy, $F_{0}$, with the variationally extremized condition,

$$
\frac{\delta \bar{F}}{\delta \Omega^{2}}=0
$$

and the stabilized condition

$$
\frac{\delta^{2} \bar{F}}{\left(\delta \Omega^{2}\right)^{2}} \geq 0
$$

The parameter $\Omega$ which renders $\bar{F}$ absolutely minimized will be chosen from the three possibilities: the non-zero solution of Eq.(11), zero and $\infty . \bar{F}$ with such an $\Omega$ is just $F_{0}$, the variational result of $F$. This procedure is essentially same as was done in Ref. [16] for Eq.(1).

Entering the above variational result into Eq.(6) and taking the logarithm, we obtain the following expression of $F$ :

$$
F=F_{0}-\frac{1}{\beta} \ln \left[<\exp \left\{-\left[S_{D}\left[\delta_{J}\right]-<S_{D}\left[\delta_{J}\right]>_{G}\right]\right\}>_{G}\right] .
$$

Now, we make a Taylor series expansion of the exponential in the last equation, and the average $<\cdots>_{G}$ can be calculated order by order through borrowing the Feynman diagram technique [14] 3 . The logarithmic operation in Eq.(13) is equivalent to discarding disconnected diagrams [14]. Consequently, we have

\footnotetext{
${ }^{3}$ Now diagrams are no longer the bare Feynman diagrams in the sense of perturbation theory owing to $\Omega \neq \omega$ which has been variationally determined.
} 


$$
F=F_{0}+\sum_{2}^{\infty} F^{(n)}
$$

with the $n$th order correction to the variational result

$$
\begin{aligned}
F^{(n)}= & (-1)^{n+1} \frac{1}{\beta} \frac{1}{n !}<\left[\left.S_{D}\left[\delta_{J}\right]\right|_{\tau=\tau_{1}}-<\left.S_{D}\left[\delta_{J}\right]\right|_{\tau=\tau_{1}}>_{G}\right] \\
& \cdots\left[\left.S_{D}\left[\delta_{J}\right]\right|_{\tau=\tau_{i}}-<\left.S_{D}\left[\delta_{J}\right]\right|_{\tau=\tau_{i}}>_{G}\right] \\
& \cdots\left[\left.S_{D}\left[\delta_{J}\right]\right|_{\tau=\tau_{n}}-<\left.S_{D}\left[\delta_{J}\right]\right|_{\tau=\tau_{n}}>_{G}\right]>_{G, C} .
\end{aligned}
$$

Here, the subscript $C$ means that only the connected diagrams have their contributions to the free energy [14]. Eq.(14) corresponds to a systematic Feynman-diagram-like expansion, and from it, one can estimate the approximate values of the free energy, $F$, order by order. Thus, we have finished the generalization of the scheme in Ref. [8] to a Bosonic case. In this scheme, the parameter $\Omega$ is variationally determined before the series expansion is performed, and it is identical for all orders. Obviously, this is a Taylor series expansion around the variational result, and so we call it the variational expansion. Because the $i$ th factor in Eq.(15) $(i=1,2, \cdots n)$ has the term $-<\left.S_{D}\left[\delta_{J}\right]\right|_{\tau=\tau_{i}}>_{G}=-\int_{0}^{\beta}<$ $-\frac{1}{2} m \Omega^{2} \delta_{J_{\tau_{i}}}^{2}+V\left[\delta_{J_{\tau_{i}}}\right]>_{G} d \tau_{i}$, which has negative sign against the major part of $F_{0}$, one can expect to get a simplified diagram rule, as will be shown for the system, Eq.(1), in the next section. Next, we apply the above procedure to the anharmonic oscillator and make a comparison with existing results in the literature.

\section{APPLICATION TO THE ANHARMONIC OSCILLATOR}

For the system, Eq.(1) $\left(V[x(t)]=\frac{1}{2} m \omega^{2} x^{2}(t)+\lambda x^{4}(t)\right)$, the procedure from Eq.(8) to Eq.(12) yields easily the variational free energy, $F_{0}$,

$$
F_{0}=\frac{1}{\beta} \ln \left(2 \sinh \left(\frac{\beta \Omega}{2}\right)\right)-\frac{3 \lambda}{4 m^{2} \Omega^{2}} \operatorname{coth}^{2}\left(\frac{\beta \Omega}{2}\right)
$$

with the variationally extremized condition $\left(\frac{\delta \bar{F}}{\delta \Omega^{2}}=0\right)$

$$
\Omega^{2}=\omega^{2}+\frac{6 \lambda}{m^{2} \Omega} \operatorname{coth}\left(\frac{\beta \Omega}{2}\right) .
$$

Here, owing to the periodicity of the path in Eq.(2), we have used the following propagator

$$
G_{\tau \tau^{\prime}}=\frac{1}{\beta} \sum_{-\infty}^{\infty} \frac{1}{m\left(\omega_{n}^{2}+\Omega^{2}\right)} e^{-i \omega_{n}\left(\tau-\tau^{\prime}\right)}=\frac{1}{2 m \Omega} \frac{\cosh \left(\frac{\beta \Omega}{2}-\Omega\left|\tau-\tau^{\prime}\right|\right)}{\sinh \left(\frac{\beta \Omega}{2}\right)}
$$

with $\omega_{n}$ the Matsubara frequency [14,15]. Eq.(16) coupled with Eq.(17) are just the variational result of $F$ in Ref. [16].

Using the relation $\frac{1}{2} m^{2}\left(\omega^{2}-\Omega^{2}\right)=-6 \lambda G_{\tau \tau}$ from Eq.(17), we have

$$
\begin{aligned}
<S_{D}\left[\delta_{J}\right]>_{G} & =\int_{0}^{\beta}<\frac{1}{2} m\left(\omega^{2}-\Omega^{2}\right) \delta_{J_{\tau}}^{2}+\lambda \delta_{J_{\tau}}^{4}>_{G} d \tau \\
& =\int_{0}^{\beta}\left[\frac{1}{2} m\left(\omega^{2}-\Omega^{2}\right) G_{\tau \tau}+3 \lambda G_{\tau \tau} G_{\tau \tau}\right] d \tau=-\int_{0}^{\beta} 3 \lambda G_{\tau \tau} G_{\tau \tau} d \tau .
\end{aligned}
$$

So, for any $i$, one has

$$
\begin{aligned}
& \left.<\left.\cdots S_{D}\left[\delta_{J}\right]\right|_{\tau=\tau_{i}}-<\left.S_{D}\left[\delta_{J}\right]\right|_{\tau=\tau_{i}}>_{G}\right] \cdots>_{G, C} \\
= & <\cdots \int_{0}^{\beta}\left[\frac{1}{2} m\left(\omega^{2}-\Omega^{2}\right) \delta_{J_{\tau_{i}}}^{2}+\lambda \delta_{J_{\tau_{i}}}^{4}-<-\frac{1}{2} m\left(\omega^{2}-\Omega^{2}\right) \delta_{J_{\tau_{i}}}^{2}+\lambda \delta_{J_{\tau_{i}}}^{4}>_{G} d \tau_{i} \cdots>_{G, C}\right. \\
=<\cdots \int_{0}^{\beta}\left[\frac{1}{2} m\left(\omega^{2}-\Omega^{2}\right)\left(\left(\stackrel{\circ}{\delta}_{J_{\tau_{i}}}\right)^{2}+G_{\tau_{i} \tau_{i}}\right)\right. & \\
& \quad+\lambda\left(\left({\stackrel{\circ}{\delta} J_{\tau_{i}}}^{4}+6 G_{\tau_{i} \tau_{i}}\left(\stackrel{\circ}{\delta}_{\tau_{\tau_{i}}}\right)^{2}+3 G_{\tau_{i} \tau_{i}} G_{\tau_{i} \tau_{i}}\right)+3 \lambda G_{\tau_{i} \tau_{i}} G_{\tau_{i} \tau_{i}}\right] d \tau_{i} \cdots>_{G, C} \\
& \quad<\cdots \int_{0}^{\beta}\left[\lambda \left(\left(\stackrel{\circ}{\delta}_{J_{\tau_{i}}}\right)^{4} \cdots>_{G, C},\right.\right.
\end{aligned}
$$


where, the symbol $\circ$ in $\stackrel{\circ}{\delta}$ means that the functional derivative with the index $i$ takes effects on $\exp \left\{\frac{1}{2} J_{\tau} G_{\tau \tau^{\prime}} J_{\tau^{\prime}} d \tau d \tau^{\prime}\right\}$ only if it makes up pair with any other functional derivative with the index $j \neq i$ to yield $G_{\tau_{i} \tau_{j}}$. In going to the second step of the last equation, a concrete analysis has led to the following equivalent properties ( $\Leftrightarrow$ means equivalence):

$$
\delta_{J_{\tau_{i}}}^{2} \Leftrightarrow\left(\stackrel{\circ}{\delta}_{\tau_{\tau_{i}}}\right)^{2}+G_{\tau_{i} \tau_{i}}, \quad \delta_{J_{\tau_{i}}}^{4} \Leftrightarrow\left(\stackrel{\circ}{\delta}_{J_{\tau_{i}}}\right)^{4}+6 G_{\tau_{i} \tau_{i}}\left(\stackrel{\circ}{\delta}_{J_{\tau_{i}}}\right)^{2}+3 G_{\tau_{i} \tau_{i}} G_{\tau_{i} \tau_{i}}
$$

In terms of the Feynman diagram language, it implies that only the legs which come from different vertices can connect each other.

Substituting Eq.(20) into Eq.(14), we can estimate the higher-order corrections to $F_{0}$ in Eq.(16) with the help of the diagram technique [14]. The free energy for Eq.(1) is now

$$
F=F_{0}-\frac{1}{\beta}<\exp \left\{-\int_{0}^{\beta} \lambda\left(\stackrel{o}{\delta}_{J_{\tau}}\right)^{4} d \tau\right\}>_{G, C}=F_{0}+\sum_{2}^{\infty} F^{(n)}
$$

with the $n$th order correction,

$$
F^{(n)}=(-1)^{n+1} \frac{1}{\beta} \frac{\lambda^{n}}{n !}<\int_{0}^{\beta} d \tau_{1}\left(\stackrel{\circ}{\delta}_{\tau_{\tau_{1}}}\right)^{4} \cdots \int_{0}^{\beta} d \tau_{n}\left(\stackrel{\circ}{\delta}_{J_{\tau_{n}}}\right)^{4}>_{G, C}
$$

Here, the modified Feynman's rules for drawing diagrams are quite simple, and they are as follows:

(1).

$$
\text { Propagator, } \quad G_{\tau_{1} \tau_{2}}
$$

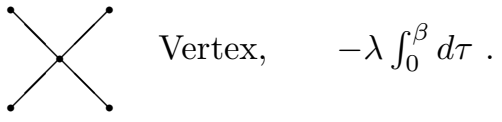

For the $n$th order, there is an additional total factor $-\frac{1}{\beta n !}$. From Eq.(23), it is evident that there will be not any Cactus diagrams appearing at any higher order, which is demonstrated by the diagrams in the next section. This simplifying feature of diagrams is similar to what occurs in the Fermionic case [8]. A further analysis indicates that there exist the following four types of building bricks for any $n$ th-order connected diagrams $(n>2)$ :

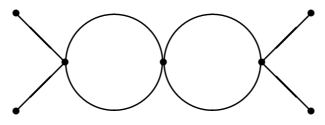

(a)

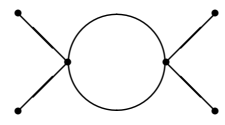

(b)

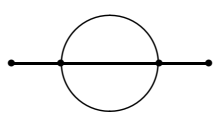

(c)

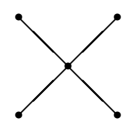

(d)

which correspond to the four kinds of partitionings of the integer "4" : (a) $2+2$, (b) $2+1+1$, (c) $3+1$ and (d) $1+1+1+1$, respectively. In this figure, the intermediate vertex of the brick (a) has two legs connected with one different vertex and the other two legs with another different vertex; the left (or right) vertex of the brick (b) has two legs connected with one different vertex and the other two legs of it will be connected with some other two different vertices respectively; the left (or right) vertex of the brick (c) has three legs connected with one different vertex and the other leg of it will be connected with some different vertex; and the vertex of the brick (d) will have its legs connected with some four different vertices respectively. These four bricks are helpful for drawing various distinct diagrams at any order as one can see from the five diagrams drawn in the next section. For example, all of them do not contain the brick (d), the second-order diagram consists of only the brick (a), so does the first diagram of the forth-order diagrams (4a).

In the next section, we calculate the free energy up to the fourth order from Eq.(22).

\section{ANALYTICAL EXPRESSIONS AND NUMERICAL RESULTS UP TO THE FOURTH ORDER}

According to the last section, the topologically non-equivalent diagrams at the second, third and fourth orders can be drawn as follows, 


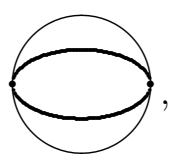

2nd

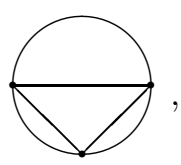

3rd

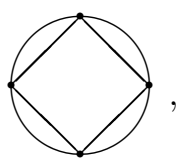

$4 \operatorname{th}(4 \mathrm{a})$

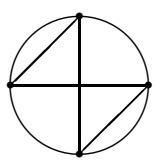

4 th $(4 b)$ and

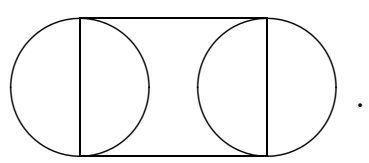

4 th $(4 \mathrm{c})$

For these five

diagrams from the left to the right, their symmetry factors (the number of topologically-equivalent diagrams appearing in the expansion) are $N_{2}=4 !, N_{3}=\frac{3 !}{3 \cdot 2} \cdot\left(2 \cdot C_{4}^{2}\right)^{3}, N_{4 a}=\frac{4 !}{4 \cdot 2} \cdot\left(2 \cdot C_{4}^{2}\right)^{4}, N_{4 b}=\frac{4 !}{4 \cdot 2} \cdot\left(C_{4}^{2} \cdot 2 \cdot C_{4}^{2}\right)^{2} \cdot 2^{4}$ and $N_{4 c}=\frac{4 !}{4 \cdot 2} \cdot\left(C_{4}^{3} \cdot 3 ! \cdot C_{4}^{3}\right)^{2} \cdot 2$, respectively. Thus, one can easily write down the corrections $F^{(2)}, F^{(3)}$ and $F^{(4)}$ according to the above diagrams and then calculate them as

$$
\begin{aligned}
F^{(2)}= & -\frac{1}{\beta} \frac{\lambda^{2}}{2 !} N_{2} \int_{0}^{\beta} d \tau_{1} d \tau_{2} G_{\tau_{1} \tau_{2}}^{4} \\
= & -\frac{3 \lambda^{2}}{64 m^{4} \Omega^{5}} \sinh ^{-4}\left(\frac{\beta \Omega}{2}\right)[6 \beta \Omega+8 \sinh (\beta \Omega)+\sinh (2 \beta \Omega)], \\
F^{(3)}= & \frac{1}{\beta} \frac{\lambda^{3}}{3 !} N_{3} \int_{0}^{\beta} d \tau_{1} d \tau_{2} d \tau_{3} G_{\tau_{1} \tau_{2}}^{2} G_{\tau_{2} \tau_{3}}^{2} G_{\tau_{3} \tau_{1}}^{2} \\
= & \frac{9 \lambda^{3}}{512 m^{6} \Omega^{8}} \sinh ^{-6}\left(\frac{\beta \Omega}{2}\right)\left\{-48+32 \beta^{2} \Omega^{2}+\left[-3+8 \beta^{2} \Omega^{2}\right] \cosh (\beta \Omega)\right. \\
& +48 \cosh (2 \beta \Omega)+3 \cosh (3 \beta \Omega)+108 \beta \Omega \sinh (\beta \Omega)\},
\end{aligned}
$$

and

$$
\begin{aligned}
F^{(4)}= & -\frac{1}{\beta} \frac{\lambda^{4}}{4 !} \int_{0}^{\beta} d \tau_{1} d \tau_{2} d \tau_{3} \tau_{4}\left\{N_{4 a} G_{\tau_{1} \tau_{2}}^{2} G_{\tau_{2} \tau_{3}}^{2} G_{\tau_{3} \tau_{4}}^{2} G_{\tau_{4} \tau_{1}}^{2}\right. \\
& \left.N_{4 b} G_{\tau_{1} \tau_{2}}^{2} G_{\tau_{3} \tau_{4}}^{2} G_{\tau_{2} \tau_{3}} G_{\tau_{2} \tau_{4}}^{2} G_{\tau_{1} \tau_{3}} G_{\tau_{1} \tau_{4}}^{2}+N_{4 c} G_{\tau_{1} \tau_{2}}^{3} G_{\tau_{3} \tau_{4}}^{3} G_{\tau_{2} \tau_{3}} G_{\tau_{4} \tau_{1}}\right\} \\
= & -\frac{3 \lambda^{4}}{32768 \beta m^{8} \Omega^{12}} \sinh ^{-8}\left(\frac{\beta \Omega}{2}\right)\left\{6291-181320 \beta^{2} \Omega^{2}+25920 \beta^{4} \Omega^{4}\right. \\
& +6\left[71+13156 \beta^{2} \Omega^{2}+2688 \beta^{4} \Omega^{4}\right] \cosh (\beta \Omega) \\
& +48\left[-134+2115 \beta^{2} \Omega^{2}+6 \beta^{4} \Omega^{4}\right] \cosh (2 \beta \Omega) \\
& -432 \cosh (3 \beta \Omega)+864 \beta^{2} \Omega^{2} \cosh (3 \beta \Omega)+141 \cosh (4 \beta \Omega)+6 \cosh (5 \beta \Omega) \\
& -191394 \beta \Omega \sinh (\beta \Omega)+129456 \beta^{3} \Omega^{3} \sinh (\beta \Omega)+42568 \beta \Omega \sinh (2 \beta \Omega) \\
& \left.+12672 \beta^{3} \Omega^{3} \sinh (2 \beta \Omega)+37750 \beta \Omega \sinh (3 \beta \Omega)+1600 \beta \Omega \sinh (4 \beta \Omega)\right\} .
\end{aligned}
$$

These analytical expressions of Eqs.(24), (25) and (26) are the main results in this section. In order to obtain them, we have had to handle the absolute value symbol in the expression of $G_{\tau \tau^{\prime}}$ (see Eq.(18)). It is straightforward to calculate the integrals in $F^{(2)}$ and $F^{(3)}$ by dividing the integration domains into 2 ! and 3! parts respectively. As for $F^{(n)}(n \geq 4)$, multi-dimensional integration domain, which exceeds our direct intuition, is involved. However, for any $n$-dimensional integration domain, one can divide it into $n$ ! sub-domains so that, for each sub-domain, the relation $\tau_{i_{1}} \leq \tau_{i_{2}} \leq \tau_{i_{3}} \leq \cdots \leq \tau_{i_{j}} \cdots \leq \tau_{i_{n-3}} \leq \tau_{i_{n-2}} \leq \tau_{i_{n-1}} \leq \tau_{i_{n}}$ holds. Then, mimicking the calculation of $F^{(3)}$, one can find the following equivalent relation

$$
\int_{0}^{\beta} d \tau_{1} \cdots \tau_{n} \Leftrightarrow \sum_{P} \int_{0}^{\beta} d \tau_{i_{n}} \int_{0}^{\tau_{i_{n}}} d \tau_{i_{1}} \int_{\tau_{i_{1}}}^{\tau_{i_{n}}} d \tau_{i_{2}} \int_{\tau_{i_{2}}}^{\tau_{i_{n}}} d \tau_{i_{3}} \cdots \int_{\tau_{i_{n-3}}}^{\tau_{i_{n}}} d \tau_{i_{n-2}} \int_{\tau_{i_{n-2}}}^{\tau_{i_{n}}} d \tau_{i_{n-1}},
$$

where the letter "P" below the summation symbol means the summation is carried out over all the $n$ ! sub-domains. Eq.(27) allows one to obtain Eq.(26) with the aid of the computer software Mathematica.

Using the above results, we can now readily calculate the free energy up to the fourth order : $F_{2}=F_{0}+F^{(2)}$, $F_{3}=F_{0}+F^{(2)}+F^{(3)}$ and $F_{4}=F_{0}+F^{(2)}+F^{(3)}+F^{(4)}$. In the following, we will numerically compare them with existing results to examine rediability of our scheme.

First, we compare our results with the exact results obtained from Ref. [6]. Using $F=-T \ln \left[\sum_{n} e^{-E_{n} / T}\right]\left(E_{n}\right.$ is the $n$th eigenenergy of Eq.(1)), letting $m=\omega=1$, and for $T<1$, one can calculate the exact free energies from 
Table V in Ref. [6]. For this case, we plot Fig.1 with $\lambda=1$. In Fig.1, the dotted, short-dashed, medium-dashed, long-dashed and solid curves are the exact free energy $F_{\text {exa }}, F_{0}, F_{2}, F_{3}$ and $F_{4}$, respectively. Fig. 1 indicates that: (i) when the temperature is near zero, $F_{2}$ and $F_{3}$ are very to close $F_{\text {exa }}$, whereas $F_{4}$ is unbounded from below; (ii) when the temperature is greater than 0.5 or so, $F_{2}$ and $F_{4}$ provide substantial corrections to $F_{0}$, and $F_{4}$ gives better than $F_{2}$ does, while $F_{3}$ is close to $F_{0}$. Here, we note that the invalidity of $F_{4}$ at very low temperature is not unexpected. Since the present scheme is basically the Taylor expansion of the free energy, the smallness of the temperature prevents and competes with the convergence process of the perturbation and finally wins over at the fourth order.

Then, we can compare our results with the accurate free energies, $F_{a c c u}$ from the Okopińska's optimized variational method [10]. In order to compare with Okopińska's data, we used the definitions of the dimensionless quantities in Ref. [10], that is, $m=1, z=\frac{1}{2} \omega^{2} \lambda^{-\frac{2}{3}}, \Omega \lambda^{-\frac{1}{3}} \rightarrow \Omega, T \lambda^{-\frac{1}{3}} \rightarrow T$ and $F_{i} \lambda^{-\frac{1}{3}} \rightarrow F_{i}$. For the case of $z=10$, which corresponds to $\lambda=0.01118$ in the dimensionlized system of Ref. [6], we give the comparison in Table I 5 . From this table, one can see that $F_{4}$ has a better agreement with $F_{a c c u}$ than $F_{2}, F_{3}$ and $F_{0}$ except for $T=30$.

Thirdly, to show the improvement of $F_{0}$ by higher-order corrections, for the range of $1<T<50$, we plotted the results in Fig. 2 to compare $F_{4}$ with $F_{0}$ in the cases of $z=0.2,1,10,30$ and 50. In Fig.2, we use the same curve type to represent $F_{4}$ and $F_{0}$, and between the curves within the same type, $F_{4}$ is always the lower. Also, Fig. 2 shows that $F_{4}$ coincides almost with $F_{0}$ for $z=30$ and 50, and the differences between $F_{0}$ and $F_{4}$ are quite large for both $z=1$ and $z=0.2$. From Fig.2, we learn that: (i) for a given temperature, with the increase of $z$, i.e., with weakening the coupling, the corrections of $F_{4}$ to $F_{0}$ get smaller; (ii) for a given $z$, with the increase of $T$, the corrections of $F_{4}$ to $F_{0}$ become larger; (iii) the quite large differences between $F_{0}$ and $F_{4}$ imply that our scheme becomes invalid with decreasing $z$ or with increasing coupling strength (for a fixed $\omega^{2}$ ). The third point is similar to the optimized expansion 9] (1994).

Finally, taking $m=\omega=1$, we compare our results with those obtained from the cumulant expansions [6] in Table II. In Table II, the free energies $F_{2}$ and $F_{3}$ are ours, the free energies $F_{1}^{K r}$ and $F_{3}^{K r}$ are the first- and third-order results from the cumulant expansion in Ref. [6] (i.e., $F_{1}$ and $F_{2}$ of Table II in Ref. [6]). The temperatures in this table is lower than 1 and the corresponding $z$ is small. So we did not include $F_{4}$ in the table due to its invalidity. This table indicates that our $F_{2}$ and $F_{3}$ are nearer to the exact value than $F_{1}^{K r}$, but is not so good as $F_{3}^{K r}$. This reflects that the convergency of our expansion is not so fast as in the cumulant expansions in Ref. [6]. As for the optimized expansions, Ref. [9] compared the free energy with the exact results in the case of both $\omega=0$ (i.e., the smallest $z$ ) and the reduced temperature less than 1, and demonstrated a fast convergence. Here, in Fig.3, our results are compared at various orders with Fig.2 in Ref. [9] (1994) 6]. In Fig.3, from the upper to lower (for large values of $\beta$ ), the first, second, sixth and seventh curves are the variational, the third-, the second- and the fourth-order results of our scheme, respectively. The third and the fourth curves are the second- and the third-order results of the optimized expansions, and the fifth curve is the exact result. The variational result is just the first-order result in the optimized expansions. From this figure, our third-order result is not so good as the results in the optimized expansions, and our second-order result is almost as good as the second-order results in the optimized expansions. This figure simultaniously indicates the invalidity of our fourth-order result. Thus, the optimized expansion has also a better convergency than our results. Additionally, the optimized expansions approach the exact result monotonously in orders, whereas our results oscillate with orders. However, we want to emphasize that our sheme is not so complicated as the optimized and cumulant expansions. The crucial difference between ours and the optimized or the cumulant expansions is that our scheme performs the variational procedure at the lowest order and, accordingly, the parameter $\Omega$ is identical for all orders, whereas in both the optimized and the cumulant expansions [9, 6], the variational procedure are performed at their truncated order, and, consequently, $\Omega$ at one order is different from the next. It is this difference that gives rise to the simplicity and slow convergency of our scheme and the fast convergency and complication of the optimized or cumulant expansions.

\section{CONCLUSION AND DISCUSSION}

In this paper, we have generalized the scheme in Ref. [8] to a Bosonic case, and taking the anharmonic oscillator, Eq.(1), as a laboratory, provided a wide test of its efficiency. Our investigations demonstrate that the present scheme can substantially improve the variational result even in the second order and when the reduced temperature is greater

\footnotetext{
${ }^{4}$ In Ref. [10], there is a typo on the rescaling expression of $T$, and here it is corrected.

${ }^{5}$ The data of the accurate free energies were provided by Okopińska, the author of Ref. 10.

${ }^{6}$ The data from the optimized expansions were also provided by Okopińska.
} 
than 0.5 or so and the reduced coupling parameter $\lambda$ is not strong (or $z$ is small), the free energy for Eq.(1) up to the fourth order in our expansion gives a good agreement with the accurate result. We also demonstrate that for the free energy of Eq.(1), when the reduced temperature approach zero, or the reduced coupling $\lambda$ is strong (or $z$ is small), the fourth-order result is invalid. Thus, from our investigations here, one can see the efficiency and limitations of our scheme. Here, we also note that the present scheme is much simpler than the optimized and cumulant expansions, albeit it is not so fast convergent as they are. We believe that a simple scheme is often necessary and useful because the non-perturbative method beyond the Gaussian approximation is extremely complicated in general. Additionally, we gave the approximate free energy of the system, Eq.(1), for moderate temperature range. Although the exact results for the moderate temperature has existed in the literature [10], our results can be readily used, as a basis of quantitative comparison, when some other approximate methods produce the free energy at the same temperature range.

In general, it should be noted that the variational perturbation theory yields an asymptotic rather than a convergent series [14], and hence a concrete range of its validity in a specific problem has no universality. As for any specific problem, the present scheme should always be used with a judicial examination of the true physical property. We believe that the present paper can provide a qualitative reference for an application of our scheme. In particular, when a specific problem is too complicated to treat beyond the Gaussian approximation with other expansions, we believe that our scheme can provide a simple and viable tool to treat it.

Finally, we want to point out that, although we treated the quantum-mechanical anharmonic oscillator only in this paper, it is straightforward to apply our method to finite temperture scalar field theory [15]. Especially, when it is generalized to the $\phi^{6}$ models [17], we expect that the simplicity of the method still holds there.

\section{ACKNOWLEDGMENTS}

Lu acknowledges A. Okopińska for providing her accurate data, and would like to thank H. S. Park for his help. This project was supported by the Korea Research Foundation (99-005-D00011). Lu's work was also supported in part by the National Natural Science Foundation of China under the grant No. 19875034.

[1] Feynman R P and Hibbs A R 1965 Quantum Mechanics and Path Integrals (New York: McGraw-Hill); Feynman R P 1972 Statistical Mechanics - a Set of Lectures, (New York: Addison-Wesley)

[2] Koehler T R 1968 Phys. Rev. 165942

[3] Seznec R and Zinn-Justin J 1979 J. Math. Phys. 201398

[4] Feynman R P and Kleinert H 1986 Phys. Rev. A 34 5080; Kleinert H 1993 Phys. Lett. A 173332 and the references therein; Okopińska A 1987 Phys. Rev. D 351835

[5] Ibañez-Meiar R, Mattingly A, Ritschel U and Stevenson P M 1992 ibid. D 45 2893; Sissakian A N, Solovtsov I L and Shevchenko O Y 1993 Phys. Lett. B 313 367; Lee G H and Yee J H 1997 Phys. Rev. D 566573

[6] Krzyweck J 1997 Phys. Rev. A 564410

[7] You S K, Jeon K J, Kim C K and Nahm K 1998 Eur. J. Phys. 19179

[8] You S K, Kim C K, Nahm K and Noh H S 2000 Phys. Rev. C 62045503

[9] Okopińska A 1987 Phys. Rev. D 36 2415; Duncan A and Jones H F 1993 ibid. D 47 2560; Vlachos K and Okopińska A 1994 Phys. Lett. A 186 375, or hep-th/9311145; 1998 Phys. Lett. A 249259

[10] Okopińska A 1987 Phys. Rev. D 361273

[11] Giachetti R and Tognetti V 1985 Phys. Rev. Lett. 55 912; 1986 Phys. Rev. B 33 7674; Wang Y R 1990 Phys. Rev. A 41 4493

[12] Kilpatrick J E and Kilpatrick M F 1948 J. Chem. Phys. 16781

[13] Bunde A and Diederich S 1979 Phys. Rev. B 19 4069; Kerr W C and Bishop A R 1986 ibid. B 34 6295; Monga M R and Pathak K N 1978 ibid. B 185859

[14] Ramond P 1990 Field Theory: a Modern Primer Revised Printing (New York : Addison-Wesley); Negele J W and Orland H 1988 Quantum Many-Particle System (New York : Addison-Wesley)

[15] Das A 1997 Finite Temperature Field Theory (Singapore : World Scientific)

[16] Haugerud H and Ravndal F 1991 Phys. Rev. D 432736

[17] Stenvenson P M and Roditi I 1986 Phys. Rev. D 33 2305; Funke M and Kümmel H G 1994 Phys. Rev. D 50 991; Lu W F, Ni G J and Wang Z G 1998 J. Phys. G 24673 
Fig.1 For the case of $T<1$ and $\lambda=1, F_{2}, F_{3}$ and $F_{4}$ the free energies up to the second, the third and the fourth orders are compared with the variational result $F_{0}$ and the exact free energy. The exact results were calculated according to $F=-T \ln \left[\sum_{n} e^{-E_{n} / T}\right]$ and Table $\mathrm{V}$ in Ref. [6. Here, $E_{n}$ represents the $n$th eigenenergy for the system Eq.(1). We took $m=\omega=1$. In this figure, when $T>0.6$, the curve for $F_{3}$ almost coincides with the curve for $F_{0}$.

Fig.2 For the case of $T>1$ and for several values of $z, F_{4}$ are compared with $F_{0}$. We use the same type of curves to represent $F_{4}$ and $F_{0}$, and the latter is always above the former. But for the cases of $z=30$ and 50, $F_{4}$ almost coincides with $F_{0}$, and for the case of $z=1.0$ and $0.2, F_{4}$ is quite lower than $F_{0}$.

Fig.3 Our results $F_{2}, F_{3}$ and $F_{4}$ are compared with the the second and third-order results by the optimized expansions which were provided by Okopińska $(\mathrm{z}=0)$. In this figure, from the upper to lower (for large values of $\beta$ ), the first, the second, the sixth and the seventh curves are the variational, the third-, the second- and the fourth-order results of our scheme, respectively. The third and the fourth curves are the second- and the third-order results of the optimized expansions respectively, and the fifth curve is the exact result.

TABLE I. Our results $F_{2}, F_{3}$ and $F_{4}$ are compared with the variational result $F_{0}$ and the accurate free energies $F_{a c c u}$ provided by Okopińska $(\mathrm{z}=10)$.

\begin{tabular}{|c|c|c|c|c|c|}
\hline $\mathrm{T}$ & $F_{4}$ & $F_{a c c u}$ & $F_{0}$ & $F_{2}$ & $F_{3}$ \\
\hline 1. & 2.262259 & 2.26225951564 & 2.262452 & 2.2622504 & 2.262261 \\
\hline 2. & 2.063913 & 2.06391575514 & 2.064409 & 2.0638734 & 2.063925 \\
\hline 3. & 1.555676 & 1.55569718863 & 1.556991 & 1.5555342 & 1.555747 \\
\hline 5. & -0.2099735 & -0.209722583045 & -0.2050294 & -0.210593 & -0.2093154 \\
\hline 10. & -7.37775 & -7.37249823358 & -7.348171 & -7.3793287 & -7.367283 \\
\hline 20. & -28.03925 & -27.9670036469 & -27.86147 & -28.0074342 & -27.92105 \\
\hline
\end{tabular}

TABLE II. Our results $F_{2}$ and $F_{3}$ are compared with the variational result $F_{0}$, the first- and third-order result, $F_{1}^{K r}$ and $F_{3}^{K r}$ obtained by the cumulant expansions ( $F_{2}$ and $F_{3}$ in Table II of Ref.[6]) and the exact free energies $F_{\text {exa }}$. In this table, $m=\omega=1$

\begin{tabular}{c|c|c|c|c|c|c|}
\hline \hline$\lambda$ & $\beta$ & $F_{0}$ & $F_{1}^{K r}$ & $F_{3}$ & $F_{3}^{K r}$ & $F_{\text {exa }}$ \\
\hline 1.0 & 5.0 & 0.812491 & 0.81188 & 0.807364 & 0.803882 & 0.803758 \\
5.0 & 5.0 & 1.244312 & 1.24353 & 1.2355 & 1.22494 & 1.22459 \\
50.0 & 5.0 & 2.54758 & 2.54675 & 2.529673 & 2.50067 & 2.49971 \\
500.0 & 10.0 & 5.425756 & 5.42536 & 5.387961 & 5.32211 & 1.216996 \\
20000.0 & 3.0 & 18.50166 & 18.5003 & 18.37314 & 18.1449 & 2.480384 \\
\hline \hline
\end{tabular}


Fig.1

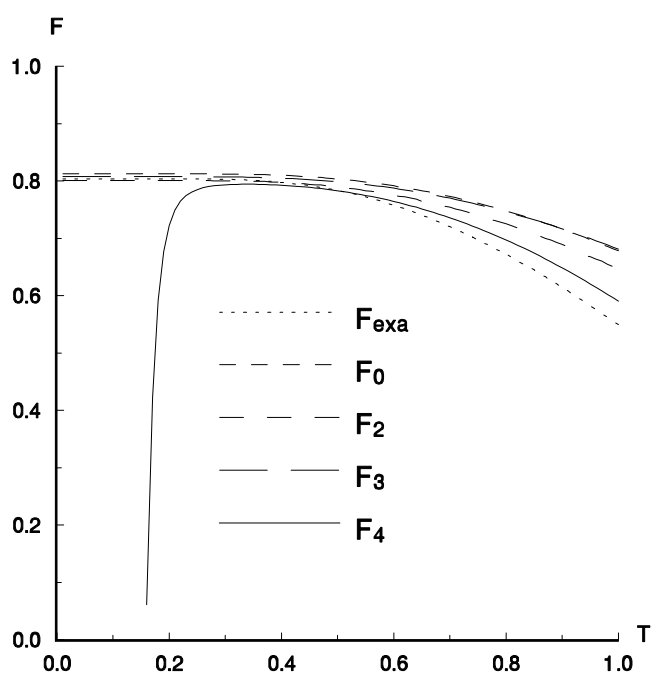


Fig.2

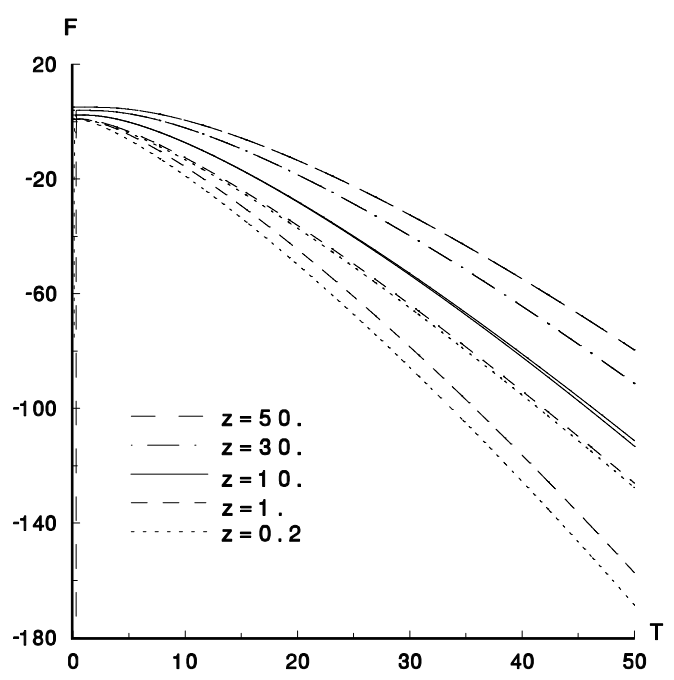


Fig.3

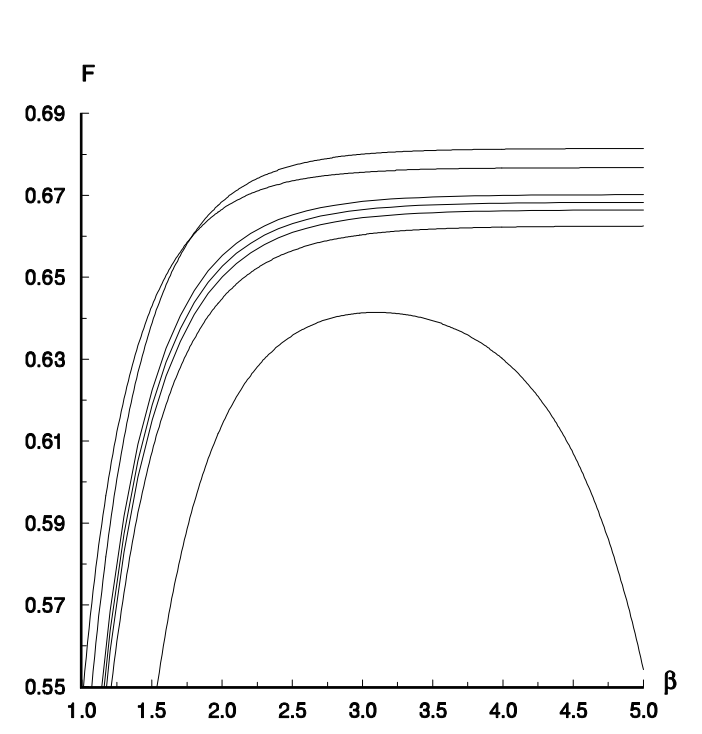

